\title{
Online Junction Temperature Cycle Recording of an IGBT Power Module in a Hybrid Car
}

\author{
Marco Denk and Mark-M. Bakran \\ Department of Mechatronics, University of Bayreuth, Universitätsstraße 30, 95447 Bayreuth, Germany \\ Correspondence should be addressed to Marco Denk; marco.denk@uni-bayreuth.de
}

Received 14 July 2014; Accepted 16 January 2015

Academic Editor: Pavol Bauer

Copyright ( 2015 M. Denk and M.-M. Bakran. This is an open access article distributed under the Creative Commons Attribution License, which permits unrestricted use, distribution, and reproduction in any medium, provided the original work is properly cited.

\begin{abstract}
The accuracy of the lifetime calculation approach of IGBT power modules used in hybrid-electric powertrains suffers greatly from the inaccurate knowledge of application typical load-profiles. To verify the theoretical load-profiles with data from the field this paper presents a concept to record all junction temperature cycles of an IGBT power module during its operation in a test vehicle. For this purpose the IGBT junction temperature is measured with a modified gate driver that determines the temperature sensitive IGBT internal gate resistor by superimposing the negative gate voltage with a high-frequency identification signal. An integrated control unit manages the $T_{J}$ measurement during the regular switching operation, the exchange of data with the system controller, and the automatic calibration of the sensor system. To calculate and store temperature cycles on a microcontroller an online Rainflow counting algorithm was developed. The special feature of this algorithm is a very accurate extraction of lifetime relevant information with a significantly reduced calculation and storage effort. Until now the recording concept could be realized and tested within a laboratory voltage source inverter. Currently the IGBT driver with integrated junction temperature measurement and the online cycle recording algorithm is integrated in the voltage source inverter of first test vehicles. Such research will provide representative load-profiles to verify and optimize the theoretical load-profiles used in today's lifetime calculation.
\end{abstract}

\section{Introduction}

The combination of an internal combustion engine and an electric machine enables the improvement of the efficiency and the performance of the drivetrain of personal cars, busses, and utility vehicles [1]. In view of the reliability and the lifetime of voltage source inverters used in hybrid-electric powertrains the IGBT power module can be considered as the most lifetime critical component. This is especially true if power modules with conventional linking and packaging technology are used. Those modules are characterized by a bond-wire connection, a direct copper bonded $\mathrm{Al}_{2} \mathrm{O}_{3}$ substrate where the chip is soldered on, and a copper baseplate. This results in a complex structure whose materials have different coefficients of thermal expansion CTE. In case of temperature cycles this CTE mismatch causes thermomechanical stresses in the modules interconnections and leads to the lift-off or the heel-cracking of bond wires or the degradation of the die-attach or the substrate solder joint [2].
To estimate the lifetime of an IGBT power module in a hybrid car a simple lifetime calculation approach has become dominant in recent years [3]. This calculation approach is derived from the lifetime estimation of mechanical parts and demands the linkage of an application typical load-profile with a lifetime model of the IGBT power module using a cycle counting algorithm and a linear damage accumulation rule. For mechanical parts like shafts or gearwheels in transmissions this lifetime calculation approach could be verified over the years and today it is possible to design their lifetime with a high accuracy. On the contrary the lifetime calculation of IGBT power modules in hybrid cars is in a very early stage and currently it is not possible to quantify the accuracy of the lifetime calculation approach. What is known, however, is that the calculation approach suffers from different factors of uncertainty like the interaction of different failure mechanisms [4] and the information loss due to the cycle counting. However, the most critical point in lifetime calculation is the limited representation accuracy of 
today's load-profiles. These theoretical profiles originate from simulation, but it is hard to consider different types of driver, different areas of operation, different hybrid strategies, and varying ambient conditions in a load-profile that is rather short in relation to the vehicle lifetime. Because of these uncertainties, there is a need to verify the theoretical loadprofiles and the lifetime calculation approach with data from field studies. For this reason this paper presents a temperature cycle recorder that can be implemented in hybrid cars to record the exposure of the IGBT power module during its real operation. The recorded load history of test vehicles or first field returns can be used to create an experience base and to optimize the load-profiles and the lifetime calculation approach of IGBT power modules. This paper shows new results of the IGBT driver and the recording algorithm and combines it with the results of the following publications [57]. In the following the state-of-the-art approach to calculate the lifetime of an IGBT power module is briefly summarized.

\section{State of the Art}

The lifetime calculation of an IGBT power module in a hybrid car requires basically the linkage of an application typical load-profile with an empirical lifetime model of the power module using a cycle counting algorithm. Today this load-profile originates from an application typical velocity profile like the New European Driving Cycle (NEDC) and the simulation of the entire hybrid-electric powertrain. A subsequent electrothermal model of the power module delivers the transient IGBT junction temperature over a certain timespan. This transient temperature curve is called the loadprofile of the power module. It is analyzed with a cycle counting algorithm and valued with an empirical lifetime model. In recent years the following lifetime models and counting algorithms have been presented.

2.1. Empirical Lifetime Models. Empirical lifetime models originate from the accelerated ageing of power modules. They specify the number of temperature cycles a power module can bear until a failure criterion is reached. In recent years various lifetime models were publicized that differ primarily in the number of parameters used to describe a temperature cycle. The elementary lifetime model is a simple Coffin-Manson law [8] that states that the number of temperature cycles to failure $N_{f}$ depends solely on the size of the amplitude $\Delta T_{J}$ of a temperature cycle:

$$
N_{f} \sim \Delta N_{J}^{\alpha} .
$$

Today there are extended lifetime models [9-11] which consider additional parameters to describe a temperature cycle. In 1997 the LESIT [9] project investigated the temperature cycle stability of power modules with conventional packaging technology from European and Japanese suppliers. It was found that the medium cycle temperature $T_{J \text {,med }}$ has a notable influence on the sustainable number of cycles. For this reason the Coffin-Manson law was extended by an Arrhenius term. Equation (2) shows the LESIT model, where the number of cycles to failure $N_{f}$ is a function of the cycle amplitude $\Delta T_{J}$ and the medium cycle temperature $T_{J \text {,med }}$. The parameters $A=640$ and $\alpha=-5$ were derived from accelerated ageing and $R=8.314 \mathrm{~J} / \mathrm{mol} \cdot \mathrm{K}$ is the gas constant and $Q=7.8$. $10^{4} \mathrm{~J} \cdot \mathrm{mol}^{-1}$ is the activation energy:

$$
N_{f}=A \cdot \Delta T_{J}^{\alpha} \cdot \exp \left(\frac{Q}{R \cdot T_{J, \text { med }}}\right) .
$$

Since the technologies of conventional IGBT power modules have been improved, in 2008 the number of sustainable temperature cycles to failure was reinvestigated by power cycling of several Infineon IGBT modules. It became apparent that many additional parameters have an impact on the module lifetime. The developed CIPS08 [10] lifetime model describes the number of cycles to failure $N_{f}$ as a function of the amplitude $\Delta T_{J}$, the minimum temperature $T_{J, \min }=$ $T_{J \text {,med }}-(1 / 2) \cdot \Delta T_{J}$, and the heating time $t_{\text {on }}$ of a temperature cycle:

$$
N_{f}=A \cdot \Delta T_{J}^{\beta_{1}} \cdot \exp \left(\frac{\beta_{2}}{T_{J, \text { min }}}\right) \cdot t_{\mathrm{on}}^{\beta_{3}} \cdot I_{B}^{\beta_{4}} \cdot V_{C}^{\beta_{5}} \cdot D^{\beta_{6}} .
$$

Moreover the current per bond wire $I_{B}$, the nominal voltage $V_{C}$, and the bond wire diameter $D$ were taken into account. The parameters $A$ and $\beta_{1}$ to $\beta_{6}$ and their validity ranges are given in [12]. For instance the heating time $t_{\text {on }}$ of a temperature cycle must be set to $t_{\text {on }}=15 \mathrm{~s}$ for $t_{\text {on }}>15 \mathrm{~s}$. Equation (3) shows the CIPS08 lifetime model. For lifetime calculation the current per bond wire can be set to $I_{B}=$ $10 \mathrm{~A}$. The diameter of the bond wire $D$ and the voltage class of the power module $V_{C}$ are constants, so that the lifetime of the power module depends solely on the temperature cycles the power module is exposed to during its operation. This comparison of different lifetime models shows that the accuracy of the empirical models used for lifetime calculation could be improved due to the more accurate specification of a temperature cycle. The present state of the art is the parameterization of a temperature cycle with its amplitude $\Delta T_{J}$, its minimum temperature $T_{J, \min }$, and its heating time $t_{\text {on }}$.

2.2. Cycle Counting Algorithm. Counting algorithms enable the evaluation of an application typical load-profile that consists of several different temperature cycles with an empirical lifetime model. For this purpose they extract and parameterize all temperature cycles within the load-profile and store them in a data vector. Widely accepted counting methods are the half-cycle counting, the maximum-edge counting, and the Rainflow counting $[13,14]$. Figure 1 shows the application of these counting algorithms on an exemplary temperature profile.

In the half-cycle counting all rising and falling edges were counted as half temperature cycles. Their amplitudes are calculated as differences of two consecutive extreme values. The minimum temperature of each cycle equals the smallest cycle temperature. This applies to all mentioned algorithms. Half-cycles with rising edges consist of a heating time, which is the time span between their occurrences. The cooling time of half-cycles with falling edges is not considered in the reviewed lifetime models, so that these cycles do 


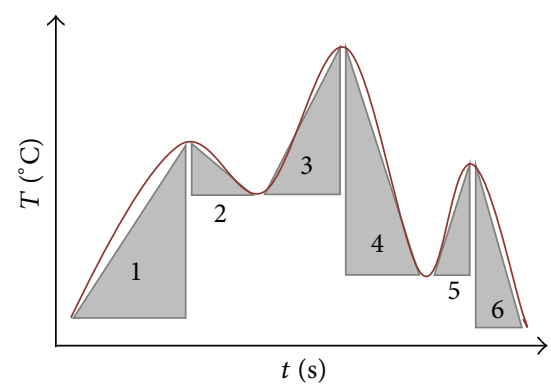

(a)

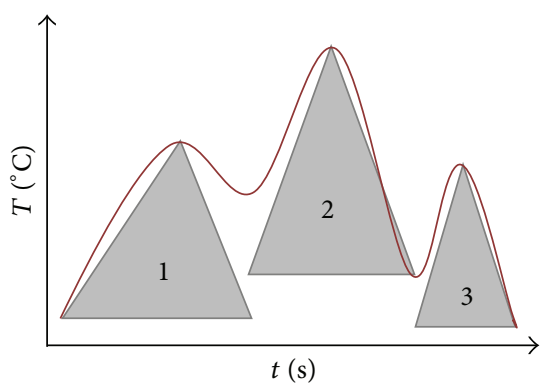

(b)

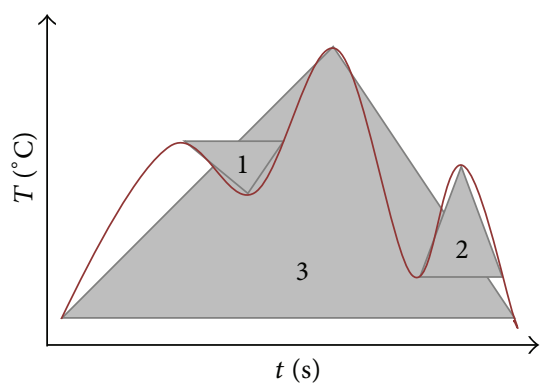

(c)

FIGURE 1: Established cycle counting algorithms: (a) half-cycle, (b) maximum-edge, and (c) Rainflow counting applied to an exemplary temperature profile.

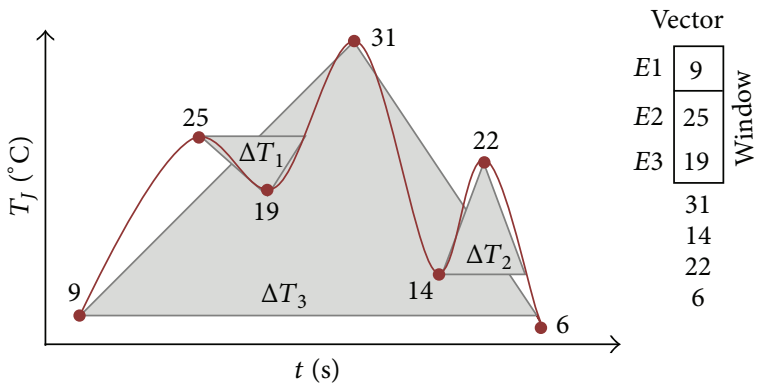

FIGURE 2: Working principle of a conventional range-counting Rainflow algorithm that scans a one-dimensional extreme value vector.

not have time stamps. Maximum-edge counting means the interpretation of the major edge of three consecutive extreme values as full temperature cycle. The heating time equals the time span between the occurrences of the first and the second extreme value.

The unique feature of the Rainflow method is the counting of closed temperature cycles, which appear as closed hysteresis curves in the stress-strain diagram [15]. This physical background distinguishes the Rainflow method from the previous described algorithms. For this reason the Rainflow algorithm became the state of the art in the lifetime calculation of power modules. The principle of the Rainflow method is standardized by ASTM E-1049 [16].

To calculate closed temperature cycles with a Rainflow algorithm the extreme values of the entire load-profile must be known. Today there are different versions of Rainflow algorithms, like fast Rainflow, range-counting, or graphical algorithms [17]. In the following the working principle of a simple range-counting Rainflow should be outlined. Figure 2 shows an exemplary temperature profile, whose extreme values are stored in a one-dimensional vector that is scanned by a moving window. A closed temperature cycle exists when the absolute difference of the extreme values E2 and E3 is greater or equals the absolute difference of the extreme values $E 1$ and E2. Otherwise, the window has to be shifted. At the starting position the extreme values in the window do not meet the cycle condition so that the window is shifted by one extreme value. The new values in the window fulfill the cycle condition and the temperature cycle $\Delta T_{1}=E 1-E 2=6^{\circ} \mathrm{C}$ is calculated. Finally the extreme values $E 1$ and $E 2$ were deleted and the scanning window is shifted back to the beginning of the vector.

Figure 3 shows the complete algorithm to convert a transient temperature profile into temperature cycles that are parameterized with their amplitudes $\Delta T_{J}$, their minimum temperatures $T_{J, \min }$, and their heating times $t_{\mathrm{on}}$. Firstly a simple three-point algorithm determines all extreme values of the load-profile and stores them with a timestamp in a two-column vector. Once all extreme values of the loadprofile were determined, the vector is scanned by the rangecounting Rainflow algorithm in search of full temperature cycles. Whenever a closed temperature cycle is found it is parameterized and stored in a three-column data vector.

On the basis of the stored temperature cycles the lifetime $L$ of the power module can be estimated according to (4), where $N_{f i}$ is the number of sustainable temperature cycles with a certain amplitude $\Delta T_{J}$, minimum temperature $T_{J \text {,min }}$, and heating time $t_{\mathrm{on}}$ and $n_{Z i}$ is the number of cycles with similar parameters that were found in the load-profile. In the case of a vector storage each temperature cycle has to be considered with $n_{Z i}=1$. The lifetime $L$ of the power module results from the linear accumulation [17] of the damage of all temperature cycles $N_{Z}$ and the scaling with the duration $t_{\mathrm{LP}}$ of the application typical load-profile. Consider

$$
L=\left(\sum_{i=1}^{N_{Z}} \frac{n_{Z i}}{N_{f i}\left(\Delta T_{J}, T_{J, \min }, t_{\mathrm{on}}\right)}\right)^{-1} \cdot t_{\mathrm{LP}} .
$$

An exemplary load-profile of a hybrid car with a duration of $t_{\mathrm{LP}}=45$ minutes incorporates $N_{E}=5487$ extreme values that were further processed in a Rainflow algorithm to $N_{Z}=\sum n_{Z i}=2743$ closed temperature cycles. The evaluation of this temperature cycles with the CIPS08 lifetime model results in a lifetime of the power module of $L=$ 8016 operating hours, that is, about one-year continuous operation. For this reason test vehicles enable the verification of a great part of the load-profile within a short period of time. It would even be possible to verify the entire lifetime calculation approach with field studies. 


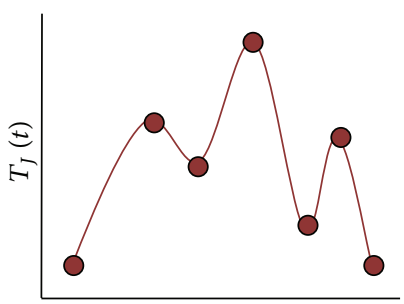

Extreme value detection algorithm

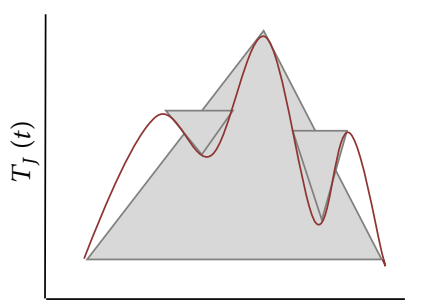

ASTM-Rainflow algorithm

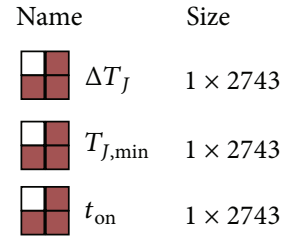

Temperature cycle storage vectors

FIGURE 3: Conventional algorithm used on desktop computers to determine and store the temperature cycles that are incorporated into the load-profile.

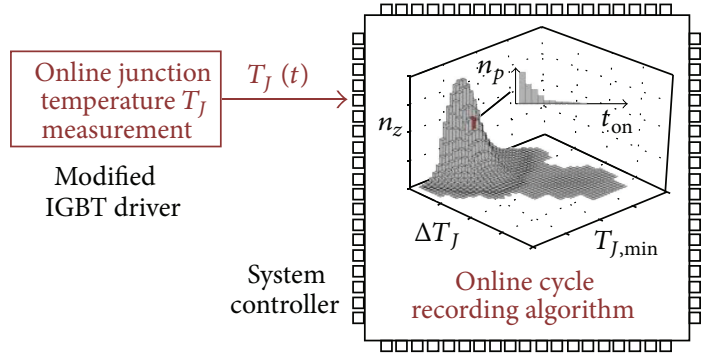

FIgURE 4: The temperature cycle recorder consists of an online cycle counting algorithm that is implemented on the system controller and a modified IGBT driver with integrated online junction temperature measurement.

\section{Temperature Cycle Recorder}

The objective of this work is the verification of the state of the art to calculate the lifetime of an IGBT power module used in hybrid-electric powertrains. Therefore the temperature cycles of the power module should be determined during the operation in the field and stored on the system controller of the voltage source inverter. Basically this requires the measurement of the IGBT junction temperature during the regular inverter operation and the online temperature cycle calculation and storage on the system controller or the IGBT driver. Figure 4 shows the block diagram of the recording concept. The junction temperature is measured with a modified IGBT driver and transmitted to the system controller with an optical fiber. The online cycle recording algorithm calculates closed temperature cycles and stores them in a compact data matrix. In the following the IGBT driver with integrated junction temperature measurement and the online cycle recording algorithm are presented in detail.

3.1. IGBT Driver with $T_{J}$ Measurement. Due to experiencing curve effects voltage source inverters used within hybrid cars contain standard IGBT power modules with conventional linking and packaging technology. The measurement of the IGBT junction temperature of those conventional power modules during their operation in a way that is suitable for series production is a very challenging task. Today's sensor concepts are based on either the installation of temperature sensors on the chip surfaces [18] or the utilization of temperature sensitive electrical parameters of the IGBT. In the laboratory the most popular temperature sensitive parameter is the saturation voltage $U_{C E}=f\left(I_{C}, T_{J}\right)$ that is measured at a constant collector current of $I_{C}=$ $100 \mathrm{~mA}$. In this case the saturation voltage has a temperature sensitivity of approximately $2.3 \mathrm{mV} / \mathrm{K}$ [19]. To realize the $U_{C E}$-method during inverter operation a switching sequence with attached measurement phase could be used [20, 21], but this also results in an interruption of the motor current and deteriorates the system properties. In $[22,23]$ the temperature sensitive quasi-threshold voltage is used for junction temperature measurement. Thereby the induced voltage across the parasitic inductance between the power emitter and an auxiliary emitter is used to trigger the measurement of the gate emitter voltage. However, the method requires an increased measurement and calibration effort and can be considered to be very noise sensitive. The feasibility of other temperature sensitive parameters for online junction temperature measurement, such as switching times [24], the current slope [25], or the width of the miller plateau [26], is rather low. Reasons for this are their small temperature sensitivity in the range of about $1 \mathrm{~ns} / \mathrm{K}$, their poor selectivity, the limited resolution of affordable sensors, and the need to add subsequent compensating procedures.

A further temperature sensitive parameter that can be found in conventional power modules, where each IGBT is built up of several paralleled IGBT single chips, is the internal gate resistor $R_{\mathrm{Gi}}$. Often this resistor is located directly under the gate bond in the center of each chip and therefore closes to the junction of the semiconductors. To use this resistor as temperature sensor in [27] a modified IGBT single chip with double-sided bond connection of the internal gate resistor is presented. This allows the impression of a small DC sense current and the determination of the temperature sensitive internal gate resistance by measuring the voltage drop across the $R_{\mathrm{Gi}}$. Although the internal gate resistor was found to be well suited for junction temperature measurement the sensor system is inappropriate for paralleled chips and nonmodified, conventional IGBT power modules.

In summary, today there is no sensor concept suitable for series production to measure the junction temperature of a conventional power module during the real inverter operation. Existing solutions either are based on the modification 


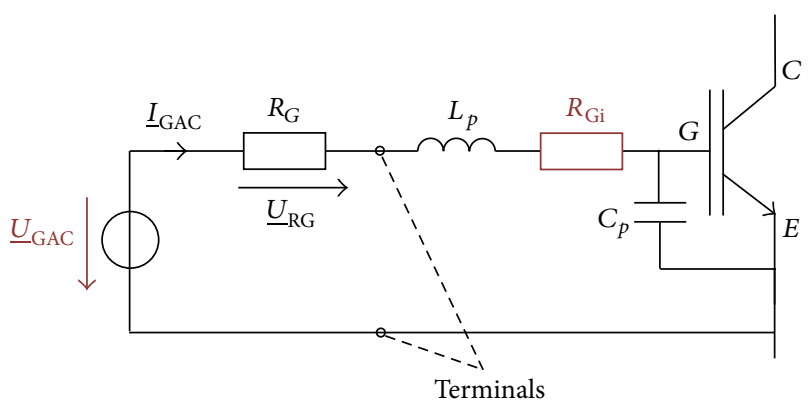

FIGURE 5: AC equivalent gate circuit of an IGBT power module consisting of the internal gate resistor $R_{\mathrm{Gi}}$ and the parasitic parameters $L_{p}$ and $C_{p}$.

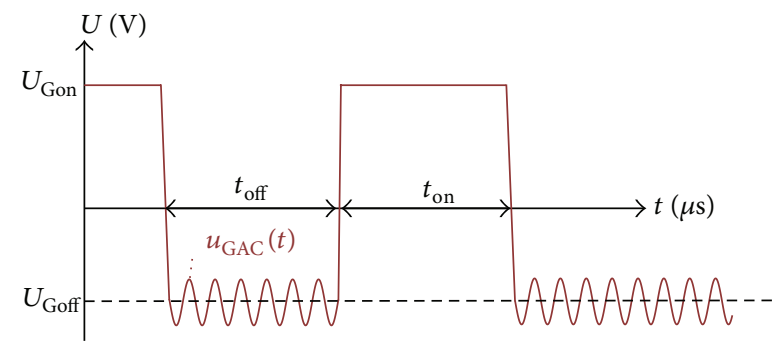

FIGURE 6: The negative gate voltage $U_{\text {Goff }}$ is superimposed with the high-frequency identification signal $u_{\mathrm{GAC}}(t)$ during the IGBT is in off-state.

of the power module or require a large intervention in the power electronic system. In the following an IGBT driver concept with integrated junction temperature measurement [5] is presented that is easy to apply, does not affect the real inverter operation, and is applicable to conventional IGBT power modules.

3.1.1. Measurement Method. To realize an easy to apply sensor system it is advantageous to integrate the junction temperature measurement in the gate circuit or in the IGBT driver, respectively. It can be seen in Figure 5 that the temperature sensitive internal gate resistor $R_{\mathrm{Gi}}$ is in series with the parasitic capacitance $C_{p}$ of the IGBT, the inductance $L_{p}$ of the gate connection, and the external gate resistor $R_{G}$. To determine the resistance of $R_{\mathrm{Gi}}$ utilizing the available terminal elements of a conventional power module the negative gate voltage is superimposed with the sinusoidal identification signal $\underline{U}_{\mathrm{GAC}}$. During the feeding in period the voltage drop $\underline{U}_{\mathrm{RG}}$ across the external gate resistor $R_{G}$ is measured. To ensure a high measurement accuracy an external gate resistor with a low temperature dependency has to be used.

Figure 6 sketches a short section of the gate voltage, where the negative voltage $U_{\text {Goff }}$ is superimposed with the sinusoidal, high-frequency identification signal $u_{\mathrm{GAC}}(t)$. To realize the measurement during the switching operation of the IGBT with a switching frequency of $f_{\mathrm{sw}}=5 \mathrm{kHz}$ the sensor response time has to be smaller than $t_{\text {off }}=100 \mu \mathrm{s}$. The frequency of the identification signal is in the range of $f_{i} \approx 5 \mathrm{MHz}$.

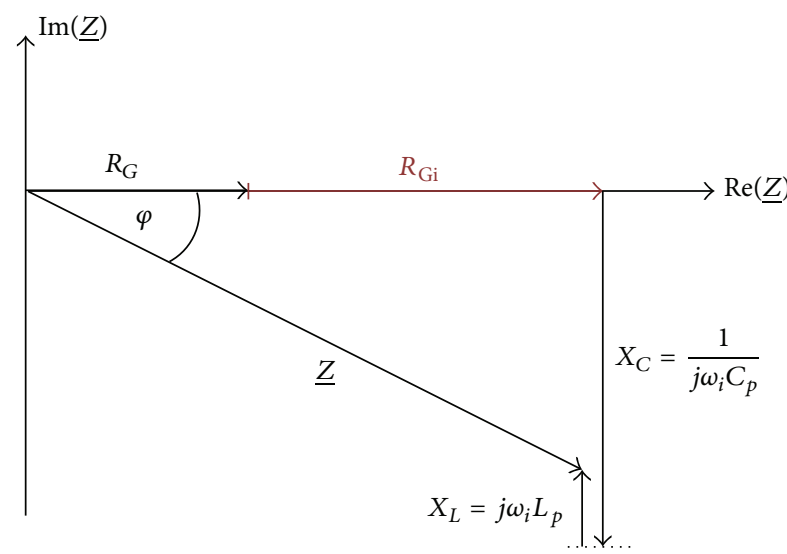

Figure 7: Vector diagram of the resistors $R_{G}$ and $R_{\mathrm{Gi}}$ and the reactances $X_{C}$ and $X_{L}$ of the parasitic components $L_{p}$ and $C_{p}$ at the frequency $\omega_{i}$.

The measurement of the amplitude $\widehat{u}_{\mathrm{RG}}$ and the phase angle $\varphi$ of the voltage drop $\underline{U}_{\mathrm{RG}}$ enables the calculation of the internal gate resistor according to (5). If the identification frequency $f_{i}$ equals the resonance frequency $f_{R}$ of the gate circuit the phase angle becomes $\varphi=0^{\circ}$. In this case the $\cos (\varphi)=1$ is constant and solely the amplitude $\widehat{u}_{\mathrm{RG}}$ of the voltage drop across the external gate resistor $R_{G}$ contains the temperature information and has to be measured. The measurement of the voltage amplitude $\widehat{u}_{\mathrm{RG}}$ can be done with a very simple sensor system. Consider

$$
R_{\mathrm{Gi}}=\frac{\underline{U}_{\mathrm{RGi}}}{\underline{I}_{\mathrm{GAC}}}=R_{G} \cdot \frac{\cos (\varphi) \cdot \widehat{u}_{\mathrm{GAC}}-\widehat{u}_{\mathrm{RG}}}{\widehat{u}_{\mathrm{RG}}} .
$$

Within a feasibility study the measurability of the internal gate resistor $R_{\mathrm{Gi}}$ in the series connection was investigated. Thereby it was assumed that the internal gate resistor can be measured if it has a sufficient large share in the total impedance $Z=|\underline{Z}|$ of the series connection. Figure 7 illustrates the external and the internal gate resistors $R_{G}$ and $R_{\mathrm{Gi}}$ as well as the reactances $X_{C}$ and $X_{L}$ of the parasitic components of the gate circuit in a vector diagram.

If the frequency $f_{i}=\omega_{i} /(2 \pi)$ of the identification signal equals the resonance frequency $f_{R}$ of the gate circuit both reactances $X_{C}=X_{L}$ distinguish themselves and the ratio of $R_{\mathrm{Gi}} / Z$ reaches its maximum value, where $Z=R_{G}+R_{\mathrm{Gi}}$. The power module $[28,29]$ used in further investigations has an $R_{\mathrm{Gi}} / Z$ ratio of $62 \%$ at a resonance frequency of about $f_{R}=$ $25.1 \mathrm{MHz}$. In the following a feeding-in method is presented that consists of a parallel path whose parasitic inductance $L_{S}$ decreases the resonance frequency to $f_{R}=4.5 \mathrm{MHz}$. The driver circuit inductance $L_{p}$ and therefore the switching behavior of the IGBT remain uninfluenced.

3.1.2. Feeding-in of the Identification Signal. The superimposition of the negative gate voltage with a high-frequency identification signal is challenging. It is especially important not to alter the switching behavior of the IGBT, to consider safety aspects, and to ensure a continuous low-resistant connection of the gate to avoid an unwanted turn-on of the IGBT. 


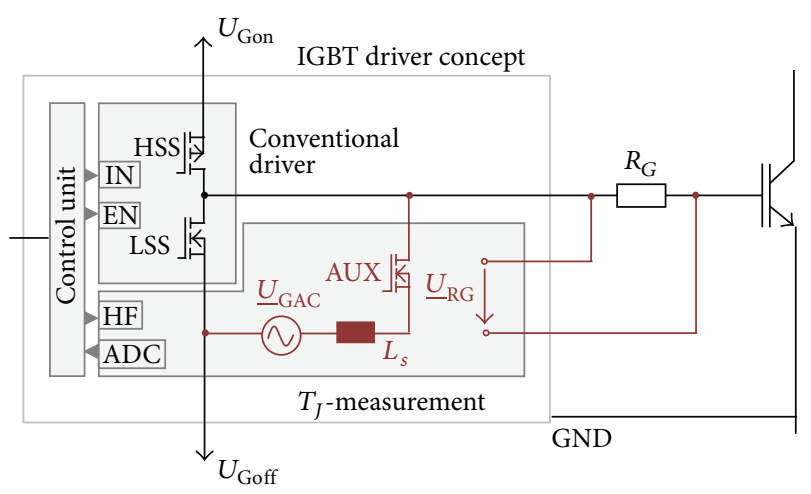

FIGURE 8: IGBT driver concept with an auxiliary MOSFET to feed in the identification voltage $\underline{U}_{\mathrm{GAC}}$. The junction temperature of the IGBT is calculated on the basis of the measured voltage drop $\underline{U}_{\mathrm{RG}}$.

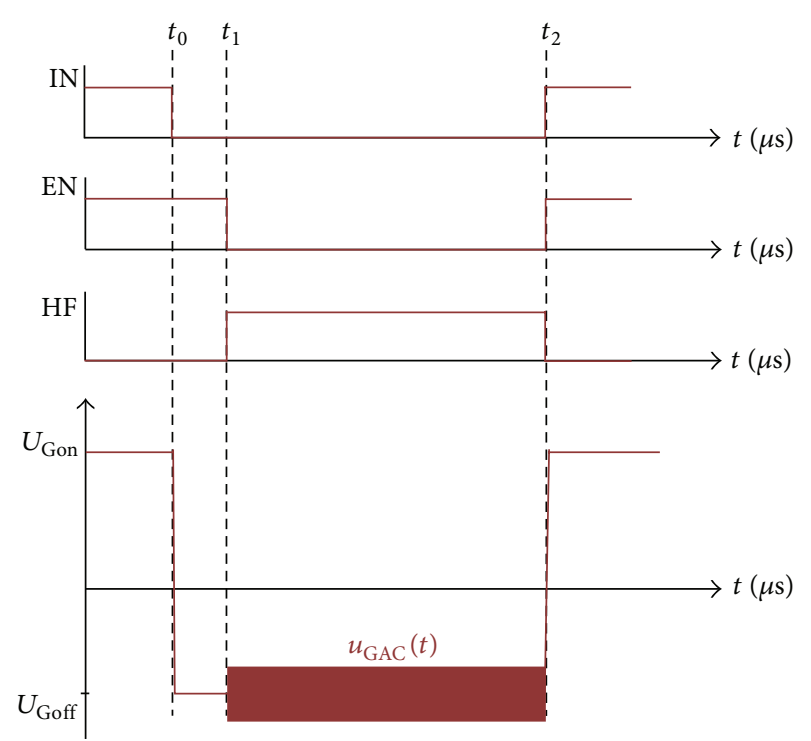

FIGURE 9: Signals to control the IGBT driver and to superimpose the negative gate voltage $U_{\text {Goff }}$ with the sinusoidal identification signal $u_{\mathrm{GAC}}(t)$.

In this context the serial feeding-in of a high-frequency signal using a transformer, whose secondary coil is integrated in series to the driver circuit, is an unsatisfactory solution [30]. On the contrary Figure 8 illustrates the parallel feeding-in of a negative gate voltage $U_{\text {Goff }}$ that is superimposed with the sinusoidal identification signal $\underline{U}_{\mathrm{GAC}}$. For this purpose a parallel auxiliary MOSFET is used. During the feedingin period this auxiliary MOSFET is turned on and the conventional IGBT driver becomes disabled so that its output is forced to a high impedance state.

Due to the parallel feeding-in the original driver circuit and thus the IGBT switching behavior remain uninfluenced. To simplify the feeding-in process during the inverter operation the driver concept consists of an integrated control unit that receives the switching pattern and transmits the measured junction temperature via an optical fiber. Figure 9

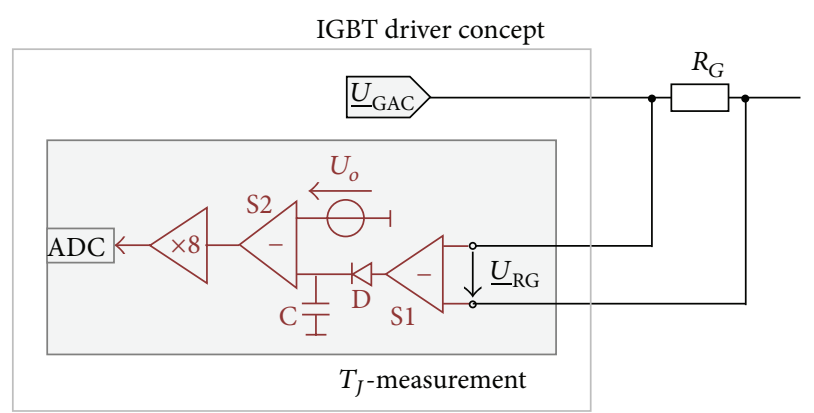

Figure 10: Circuit to measure the temperature dependent amplitude of the voltage drop $U_{\mathrm{RG}}$ and to convert it into an $\mathrm{ADC}$ compatible direct voltage.

sketches the control signals to feed in the identification voltage $u_{\mathrm{GAC}}(t)$ during the switching operation.

Initially the IGBT driver is enabled EN $=1$ and its highside-switch (HSS) is conductive (IN =1), so that the positive voltage $U_{\mathrm{Gon}}$ is applied at the gate and the IGBT is in on-state. At the time $t_{0}$ the IGBT is switched off through the negative gate voltage $U_{\mathrm{Goff}}$ that is applied by the low-side-switch (LSS) by setting $\mathrm{IN}=0$. To apply the negative, superimposed gate voltage $u_{\mathrm{GAC}}$ at the time $t_{1}$ the IGBT driver is disabled, setting $\mathrm{EN}=0$, so that its output is forced to a high impedance state. At the same time the feeding-in of the identification voltage is activated by setting $\mathrm{HF}=1$. To sustain a lowresistant gate connection the signal voltage source $\underline{U}_{\mathrm{GAC}}$ incorporates an impedance converter with a very low output impedance. During the feeding-in of the identification signal the measurement setup is analyzing the voltage drop $\underline{U}_{\mathrm{RG}}$ to generate an $\mathrm{ADC}$ compatible sensor voltage. At the time $t_{2}$ the driver concept received the instruction to reactivate the IGBT. Therefore the identification voltage is disabled $(\mathrm{HF}=0)$ and the positive gate voltage is applied, setting EN $=1$ and IN $=1$. In the case of failure the current limited buffer becomes deactivated, so that the gate signal of the IGBT driver is dominant.

3.1.3. Generation of the Sensor Output Voltage. As it was outlined earlier, in case of resonance the amplitude of the voltage drop $\underline{U}_{\mathrm{RG}}$ across the external gate resistor $R_{G}$ depends linearly on the junction temperature of the IGBT. In Figure 10 a measurement setup is presented that converts the amplitude change into an ADC compatible direct voltage. Therefore the voltage drop across $R_{G}$ is picked up differentially with the subtractor S1, rectified with the Schottky diode $D$, and smoothed by the capacitor $C$. The level of the resulting direct voltage changes upon a certain offset linearly with the junction temperature. To adjust the voltage change to the input voltage range of an $\mathrm{ADC}$, as a first step, the offset voltage $U_{0}$ is subtracted in a way that the ADC input voltage equals $U_{\mathrm{ADC}}=1 \mathrm{~V}$ at an IGBT junction temperature of $T_{J}=20^{\circ} \mathrm{C}$. Later, this offset voltage is used for the calibration of the junction temperature measurement.

Secondly, an operational amplifier adjusts the ADC input voltage to be $U_{\mathrm{ADC}}=3 \mathrm{~V}$ at $T_{J}=120^{\circ} \mathrm{C}$. This results in 


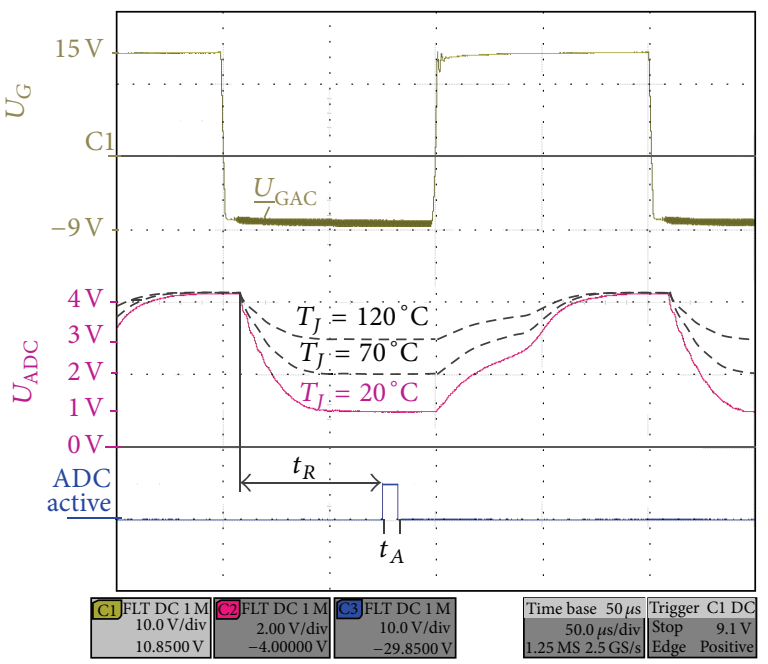

FIGURE 11: Measurement result of the driver concept where the identification voltage $\underline{U}_{\mathrm{GAC}}$ leads to a temperature dependent sensor voltage $U_{\mathrm{ADC}}$ that is sampled by an analog to digital converter during the time $\operatorname{span} t_{A}$.

a temperature sensitivity of $20 \mathrm{mV} /{ }^{\circ} \mathrm{C}$. When using a 10 bit analog to digital converter with a reference voltage of $5 \mathrm{~V}$ the junction temperature can theoretically be measured with a resolution of $0.24^{\circ} \mathrm{C} / \mathrm{bit}$. The response time of the measurement setup to generate a valid sensor voltage is primarily defined by the capacitor $C$ and is set to $t_{R}<70 \mu \mathrm{s}$.

3.1.4. Experimental Results. To qualify the presented measuring method a prototype of the IGBT driver with junction temperature measurement was developed and integrated in the double pulse experiment with $U_{C E}=600 \mathrm{~V}$ and $I_{C}=$ $250 \mathrm{~A}$. A tempering system enables the homogenous heating of the power module to different junction temperatures $T_{J}$. Figure 11 shows the gate voltage $U_{G}$ at a switching frequency of $f_{\text {sw }}=5 \mathrm{kHz}$. After the IGBT is switched off the negative gate voltage $U_{\mathrm{Goff}}$ is superimposed with the identification signal $\underline{U}_{\mathrm{GAC}}=0.6 \mathrm{~V}$ with a frequency of $f_{i} \approx 4.5 \mathrm{MHz}$. The sensor output voltage $U_{\mathrm{ADC}}$ reaches, after a response time of $t_{R} \approx 70 \mu \mathrm{s}$, a constant voltage level that is sampled by the analog to digital converter of the control unit during the timespan $t_{A} \approx 6 \mu \mathrm{s}$. It can be seen that the sensor output voltage increases linearly with an increasing junction temperature.

To determine the calibration curve of the driver concept the sensor output voltage was measured at four different junction temperatures. At each temperature level the sensor output voltage was measured several times to determine the standard deviation and the remaining noise of the temperature measurement. The measurement result in Figure 12 shows that the sensor output voltage $U_{\mathrm{ADC}}$ is a very linear function of the junction temperature $T_{J}$. The standard deviation of the voltage measurement was found to be $\sigma_{\mathrm{UADC}} \leq 24 \mathrm{mV}$ that corresponds to a remaining noise of the temperature measurement of less than $\pm 1.0 \mathrm{~K}$. To realize an easy to apply IGBT driver, this calibration curve is programmed on the

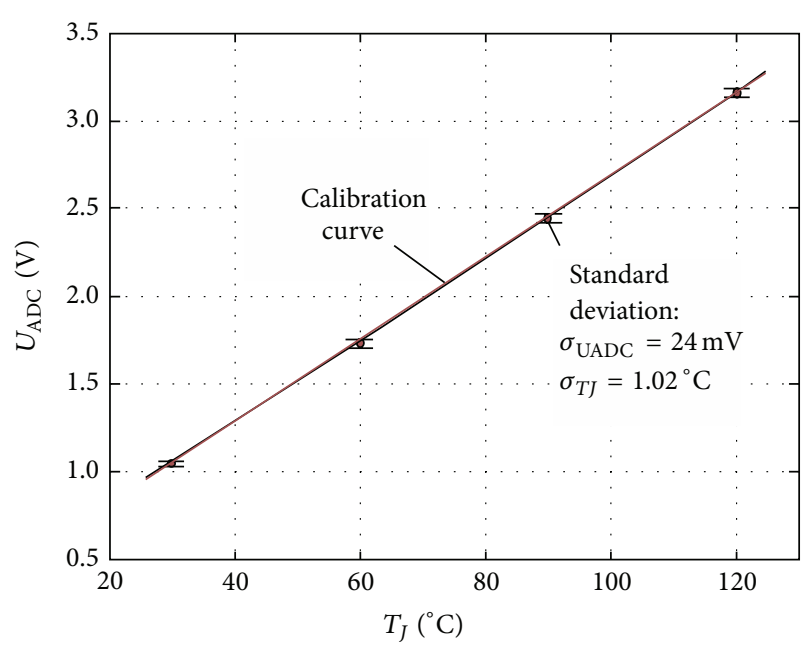

FIGURE 12: Calibration curve of the IGBT driver with junction temperature measurement.

control unit of the driver concept, so that the measured sensor output voltage can be translated immediately in the junction temperature of the IGBT and transmitted to the system controller of the voltage source inverter with an optical fiber. Because of process variations of the internal gate resistor the IGBT driver has to be calibrated at a known junction temperature, for example, at room temperature. Thereby the offset voltage $U_{0}$ is set in a way that the sensor output voltage at a homogeneous calibration temperature complies with the reference calibration curve on the control unit. The automatic calibration process is organized by the control unit and enables the pairing of the IGBT driver to a power module that is still installed in the voltage source inverter.

To qualify the IGBT driver during the regular inverter operation a test setup consisting of one inverter phase that is connected to a virtual electric machine was developed. All IGBTs were controlled by a dSpace system. A MATLAB Simulink model allows the emulation of different loadprofiles of a hybrid-electric powertrain. During the regular switching of the IGBTs the developed IGBT driver measures the junction temperature of one IGBT and reports it to the $\mathrm{d}$ Space system, where it is displayed in real time. To verify the temperature measurement with an IR camera a coated power module was used. Figure 13 shows the motor phase current $\underline{I}_{\mathrm{AC}}$ and the junction temperature $T_{J}$ that is measured with the developed IGBT driver $T_{J, D R}$ and an IR camera $T_{J, I R}$. The timespan of the load-profile is one minute. It can be seen that the temperature variations measured with the IGBT driver are in a very good agreement with those obtained with the IR camera. For this reason the developed IGBT driver is suitable for the temperature cycle recording of a power module during the inverter operation in a hybrid car.

Figure 14 shows the temperature profile of the IGBT for a timespan of 45 minutes. It can be seen that the loadprofile consists of a majority of operational temperature cycles caused by the transient power flow over the power module. Those active temperature cycles depend on the driver command and the superior hybrid strategy and lead 

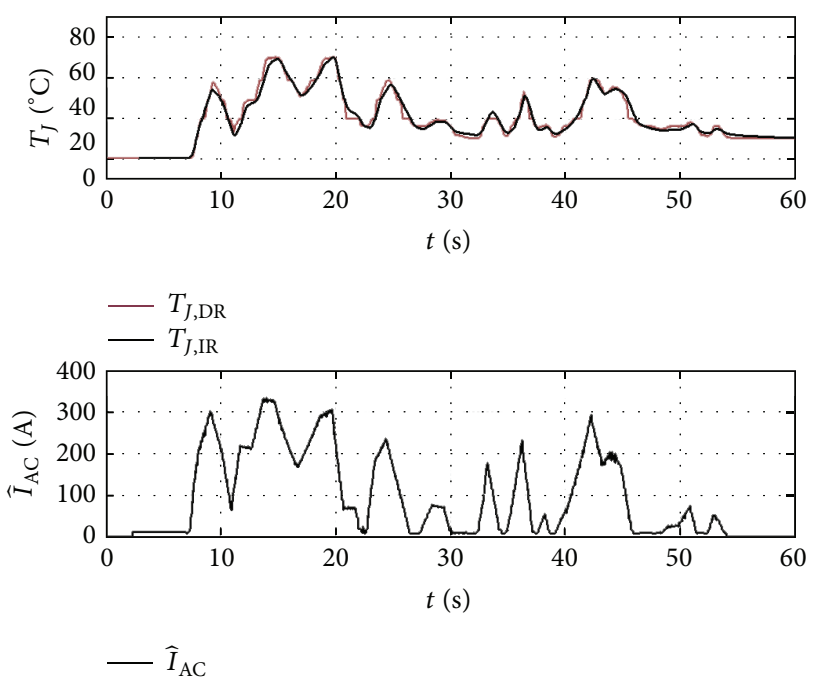

FIGURE 13: Junction temperature during inverter operation measured with the IGBT driver and an IR camera.

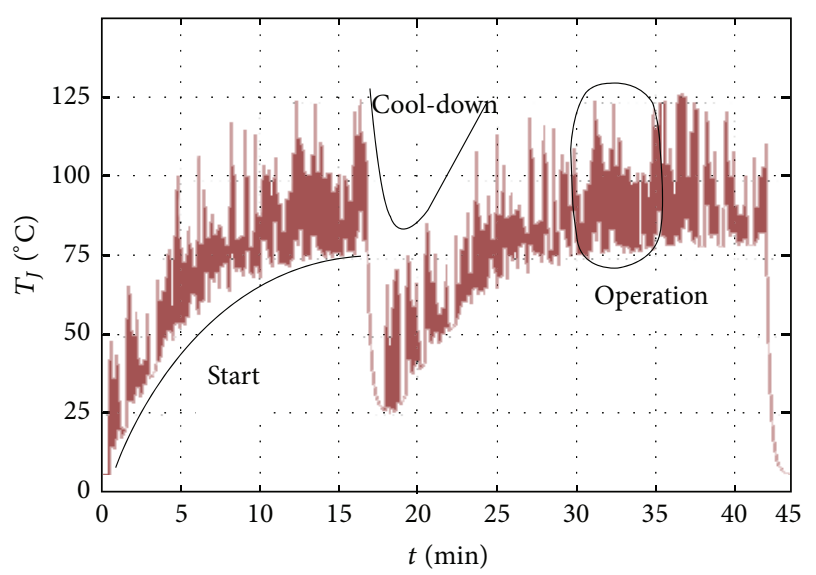

FIGURE 14: Typical load-profile of an IGBT power module in a hybrid car that specifies the IGBT junction temperature during a mission of 45 minutes.

to the heating of the coolant and the cold plate temperature during the first 15 minutes of the driving. The cool-down in the middle of the load-profile represents a time period without electric power flow. Consequently the coolant and the power module temperature decline until the subsequent operation cycles will cause the reheating of the system. After about 42 minutes the mission ends and the entire system cools down. In view of the homogeneous temperature variation of the power module during the start and the cooldown, these cycles should be labeled as passive ones.

Because of the limited storage capacity on the system controller it is not possible to store the whole temperature profile over a representative long period of time. For this purpose, an online recording algorithm is presented that processes the measured junction temperature into a compact set of information that is relevant for the verification of the theoretical load-profiles and the lifetime calculation approach. In view of the state of the art the verification can be done on the basis of temperature cycles that were calculated with a Rainflow algorithm and stored with their amplitude, their minimum temperature, and their heating time.

3.2. Online Temperature Cycle Recording Algorithm. The online calculation and storage of temperature cycles on the system controller require a modification of the state-of-theart algorithms used on desktop computers with regard to their online ability, their computing power, and the required storage capacity. Thereby the overall objective is to reach a compromise between a minimum calculation and storage effort and a maximum accuracy regarding lifetime relevant information. Figure 15 shows the block diagram of the developed online cycle recording algorithm that consists of an extreme value filter algorithm, an online Rainflow algorithm, and a classified storage of the determined temperature cycles in a four-dimensional frequency distribution. In the following the working principles of the algorithms are presented.

3.2.1. Extreme Value Filter Algorithm. The calculation of temperature cycles using a cycle counting algorithm requires previous identification of extreme values. Therefore a threepoint algorithm [31] with a sliding average filter can be used. The filter averages the temperature profile and enables the filtering of noise. It is not suitable to filter out small temperature variations in the range of $\Delta T_{J} \approx 5^{\circ} \mathrm{C}$, as they were caused by the hybrid strategy. The three-point algorithm picks out three subsequent temperature values. If the second value is higher than the first and third value a maximum is identified. Because of the limited filter level this algorithm finds a lot of extreme values, which have to be further processed in the online Rainflow. To reduce the computing power a logical filter algorithm was developed that enables the filtering of these small temperature variations but still delivers the exact temperature of valid extreme values. The principle of the filtering is outlined in Figure 16. In the filter routine two variables $T_{S}$ and $t_{s}$ were increased with the temperature $T_{J}(t)$. Only after the temperature declines, a predefined filter temperature $T_{F}$, a valid extreme value is detected. It occurred at the time $t_{s}$ and has the temperature value $T_{S}$.

In Figure 17 the filter algorithm is applied to a cutout of the presented load-profile of a hybrid car. The filter temperature is set to $T_{F}=5^{\circ} \mathrm{C}$. It can be seen that the loadprofile consists of many small temperature variations, which does not contribute notably to the damage of the power module. These temperature variations are smaller than the specified filter temperature so that they are not identified as valid extreme values. In a sequence of small temperature variations the logical filter algorithm determines the lowest extreme value. In this view the developed extreme value filter meets all requirements and combines an adjustable filter level and a high data reduction but still delivers the accurate temperature of valid extreme value.

The filter temperature should be set in a way that it enables the highest possible data reduction without the rejection of lifetime relevant information. To parameterize the filter for its use in a hybrid car Figure 18 shows the number of identified 


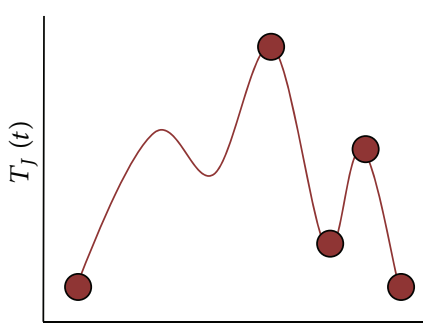

Extreme value filter algorithm

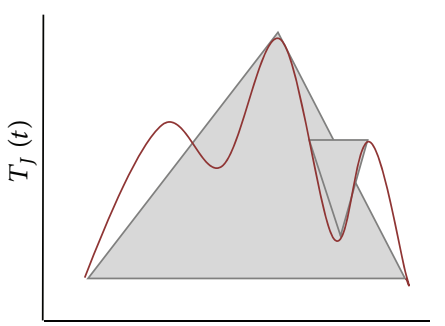

Online cycle counting algorithm

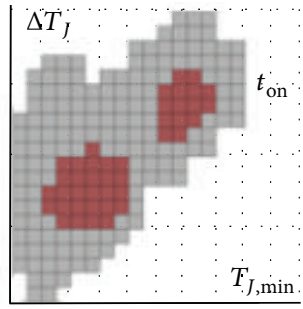

4D-frequency

distribution

FIGURE 15: Online cycle counting algorithm to calculate and store temperature cycles with less effort during the operation of the IGBT power module.

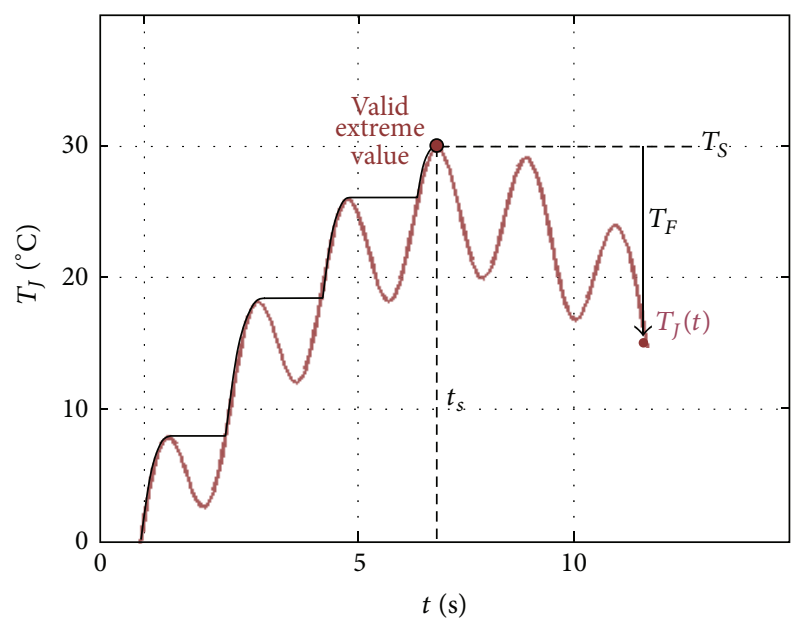

FIGURE 16: Working principle of the extreme value filter algorithm with the auxiliary variables $T_{S}$ and $t_{s}$ and the adjustable filter temperature $T_{F}$.

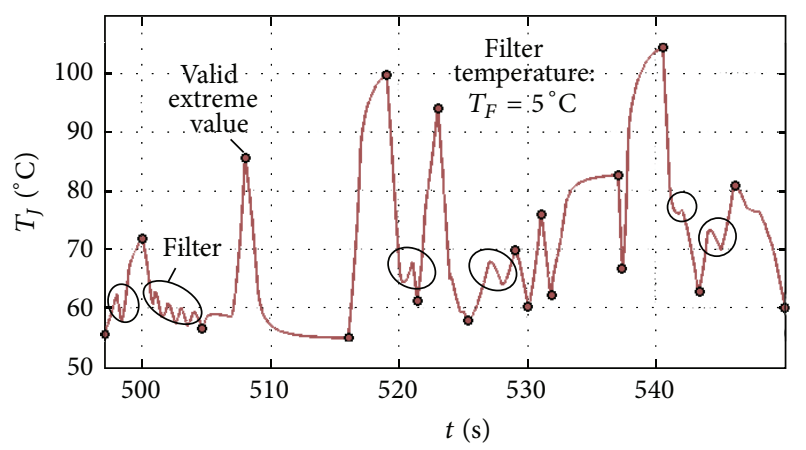

FIGURE 17: Application of the extreme value filter algorithm with a filter temperature of $T_{F}=5^{\circ} \mathrm{C}$ to a cutout of the load-profile of a hybrid car.

extreme values $N_{E}$ in the load-profile as a function of the filter temperature $T_{F}$. Moreover the error $E$ in the calculated lifetime due to the filtering of small temperature variations is diagrammed.

It appears that a filter temperature of $T_{F}=5^{\circ} \mathrm{C}$ leads to a significant reduction of the number of extreme values by

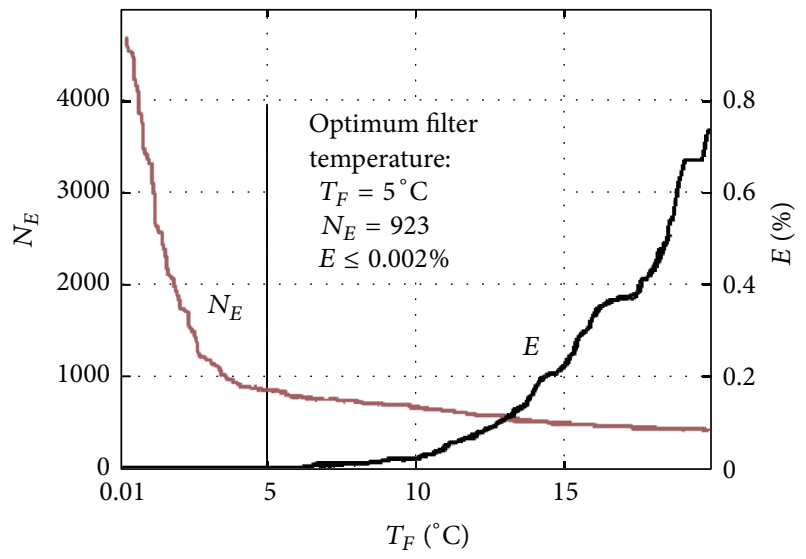

FIgURE 18: The number $N_{E}$ of extreme values that were found in the presented load-profile of a hybrid car decreases with an increasing filter temperature $T_{F}$.

more than 85 percent. The filtering of the small temperature variations that are caused by the hybrid strategy results in a deviation of the calculated lifetime of $E=+0.002$ percent. In view of the major reduction of extreme values and therefore the required computing power, this minor calculation error is acceptable. Consequently the optimum filter temperature to analyze the presented load-profile of a hybrid car is set to $T_{F}$ $=5^{\circ} \mathrm{C}$.

Finally the result of the filtering in the lifetime calculation should be demonstrated. Therefore Figure 19 shows the number $n_{Z}$ of temperature cycles with similar amplitudes $\Delta T_{J}$ that were determined with a filter level of $T_{F}=0^{\circ} \mathrm{C}$ and $T_{F}=5^{\circ} \mathrm{C}$. Additionally the lifetime consumption due to the temperature cycles of each class was calculated with the CIPS08 lifetime model and normalized to one operating hour $\mathrm{LC} / \mathrm{h}$. It is obvious that cycles with amplitudes of $\Delta T_{J} \leq 5^{\circ} \mathrm{C}$ make up a majority of all cycles but do not contribute notably to the lifetime consumption of the power module, so that the accumulated lifetime consumption is nearly identical.

In summary the filter algorithm prevents the calculation of temperature cycles that are smaller than a filter temperature of $T_{F}=5^{\circ} \mathrm{C}$, so that the computing power of the online Rainflow algorithm can be significantly reduced. In Figure 19 all greyed temperature cycles with $\Delta T_{J} \leq T_{F}$, which make up 


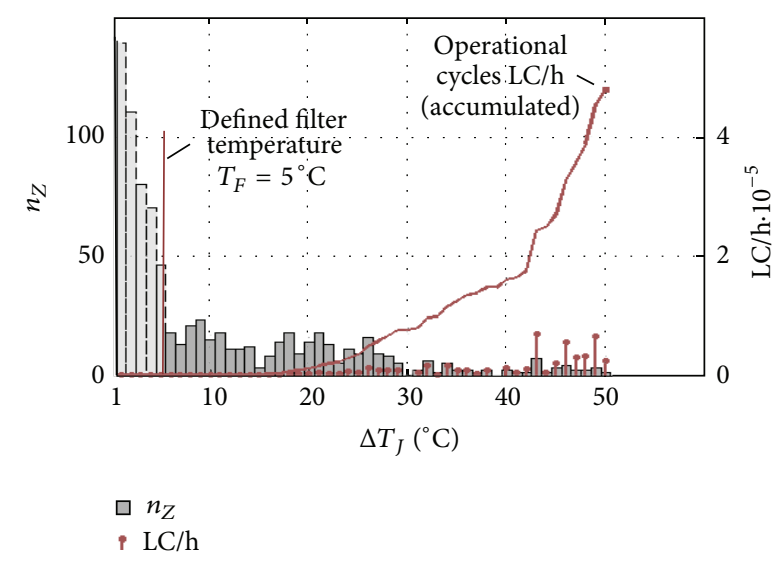

FIgURE 19: Number $n_{Z}$ of temperature cycles $\Delta T_{J}$ determined with the filter temperatures $T_{F}=0^{\circ} \mathrm{C}$ and $T_{F}=5^{\circ} \mathrm{C}$ and a subsequent Rainflow algorithm.

a share of about 85 per cent, will not be calculated anymore. Despite the filtering the exact temperature of valid extreme values is determined.

3.2.2. Online Cycle Counting Algorithm. The ability of counting algorithms to calculate cycles during the inverter operation depends on their working principle. As it was illustrated in Figure 1 the half-cycle or the maximum-edge method calculates a temperature cycle on the basis of two or three subsequent extreme values. For this reason the online implementation of those simple counting methods would be rather simple. Online Rainflow algorithms are described in [3234]. The feature of the Rainflow method is the counting of closed temperature cycles. For this reason an online Rainflow always demands the intermediate storage of extreme values in a working storage, so that its online implementation on the system controller is complicated.

Substitutability of the Rainflow Method. In the following investigations it should be analyzed whether the Rainflow method can be substituted with an easy to implement halfcycle or maximum-edge algorithm [6]. For this purpose the capability of the three algorithms to convert the presented load-profile into a distribution of defined temperature cycles is investigated. Figure 20 shows the number of temperature cycles $N$ with similar amplitudes $\Delta T_{J}$ that were extracted with a Rainflow algorithm. Beside the majority of operational temperature cycles with amplitudes of $\Delta T_{J}<70^{\circ} \mathrm{C}$ two passive cycles with amplitudes of $\Delta T_{J}>100^{\circ} \mathrm{C}$ were calculated. These cycles are caused by the homogenous heating of the entire power module during the start and the cool-down. All cycles were valued with the CIPS08 lifetime model and normalized to their lifetime consumption per operating hour $\mathrm{LC} / \mathrm{h}$. It turns out that the lifetime consumption of the two passive cycles is greater than the accumulated lifetime consumption of all operational cycles. In this view it is very important to extract and parameterize passive cycles with a high accuracy. Because of its physical background the results of the Rainflow

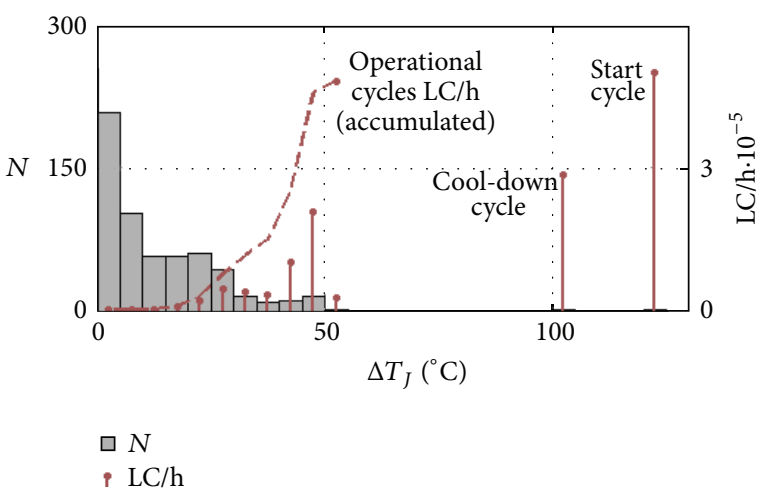

FIGURE 20: Number of temperature cycles $N$ with similar amplitudes $\Delta T_{J}$ that were determined with a Rainflow counting algorithm. The cycles were valued with the CIPS08 lifetime model; $\mathrm{LC} / \mathrm{h}=$ $f\left(\Delta T_{J}, T_{J, \min }, t_{\mathrm{on}}\right)$.

counting should be taken as reference for the evaluation of half-cycle and maximum-edge counting.

The half-cycle method extracts twice as many operational cycles. Their valuing as half temperature cycles leads to a reduced operational lifetime consumption compared to the Rainflow algorithm [6]. This applies equally to the passive cycles. Additionally the passive cycle amplitudes were found significantly smaller than in the Rainflow counting, so that their lifetime consumption is drastically undervalued. The maximum-edge counting compensates the undervaluing of the passive cycles partially due to the interpretation of the half temperature cycles as full ones. Nevertheless the cycle amplitudes differ from the Rainflow counting and cause a smaller lifetime consumption.

In summary, simple counting methods calculate a significant lower lifetime consumption than the Rainflow method so that they predict a longer lifetime of the power module. In this view it is not possible to substitute the Rainflow method with a simple counting algorithm and there is a need to implement an online Rainflow.

Online Rainflow Algorithm. For the calculation of temperature cycles with a state-of-the-art Rainflow algorithm all extreme values of the load-profile have to be stored in a vector. In the case of longer test drives this also results in a large quantity of data that has to be stored temporarily in a working storage. Contrarily to the presented rangecounting Rainflow that scans the working storage with a window in search of closed temperature cycles Figure 21 shows an online version of this principle. Thereby the window is fixed at the beginning of a working storage with a variable size. Once a new extreme value appears the working storage is shifted and the new extreme value is written to the E3 position. The Rainflow cycle condition is checked and if it is true the temperature cycle is calculated and exported to the storage. The accompanied extreme values were deleted and the working storage is shifted for two positions. Since one new extreme value can close more temperature cycles the Rainflow cycle condition is checked again until it becomes false. To calculate the cycle heating times a corresponding 


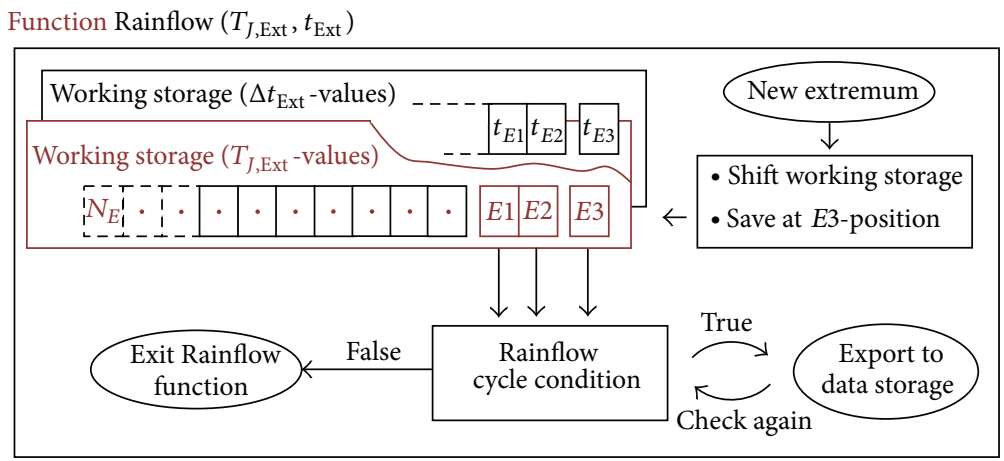

FIGURE 21: Working principle of the online Rainflow algorithm that consists of a variable working storage for extreme values and their time information and a routine to check the Rainflow cycle condition $(|E 2-E 3| \leq|E 2-E 1|)$.

working storage for the time stamps of the extreme values is available. To enable online cycle recording for an unlimited driving time with a storage capacity of a few bytes only the timespans between the extreme values were stored. This enables a reconstruction of heating times of up to 255 seconds that is enough compared with the maximum specified heating time of the CIPS08 model.

Working Storage Overflow. A critical parameter of an online Rainflow algorithm that should be implemented on a microcontroller is the size of the required working storage. In the following the number of extreme values in the working storage during the analysis of the load-profile should be examined. Therefore Table 1 outlines four exemplary temperature profiles $T_{J}(t)$, the number of extreme values $N_{E}$ in the working storage, and the extracted temperature cycles with the amplitudes $\Delta T_{J}$. It becomes clear that solely the temperature profile 3 causes an increase of the number of extreme values in the working storage above the overflow level of $N_{E \text {,max }}=10$. After the start temperature is reached all cycles were closed simultaneously and the working storage is emptied. All other temperature profiles enable the instantaneous calculation of cycles and do not increase the number of extreme values in the storage.

In a hybrid car temperature profile 3 typically arises during the voltage- and frequency-controlled starting procedure of the electric machine [35]. Figure 22 sketches an exemplary junction temperature profile during the start-up of the electric machine in a hybrid car. It consists of many small temperature cycles, whose amplitude and frequency depend on the size and the fundamental frequency of the inverter output current.

The oscillations of the junction temperature during the start-up of the electric machine cause an increase of the number of extreme values $N_{E}$ within the working storage. This leads to an overflow of the available working storage with a maximum size of 17 extreme values and a loss of information. To avoid an overflow the developed online Rainflow algorithm was expanded by an overflow routine, which is scanning the working storage in search of the starting point of profile 3 once the overflow level is reached. Subsequently all temperature cycles of the detected sequence

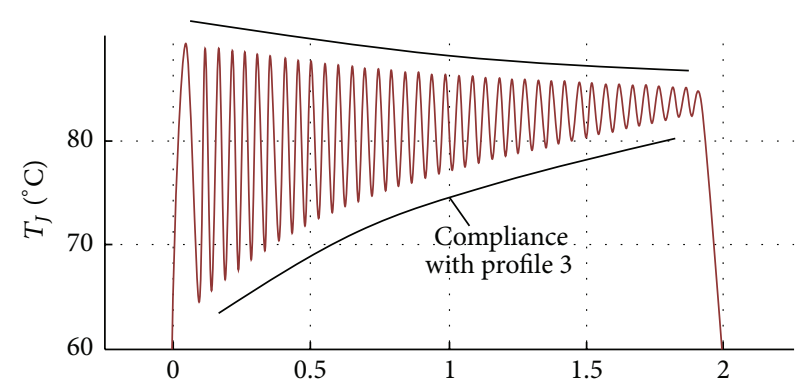

Figure 22: The junction temperature profile during the start-up of the electric machine complies with the exemplary temperature profile number 3 .

were calculated and deleted from the working storage. For this reason the overflow routine allows the use of a small working storage and improves the implementation of the Rainflow counting on a microcontroller.

3.2.3. Temperature Cycle Storage. In view of the state of the art the lifetime calculation on the basis of the presented load-profile demands the storage of each cycle with its amplitude $\Delta T_{J}$, its minimum temperature $T_{J \text {, min }}$, and its heating time $t_{\text {on }}$ [6]. To store the calculated temperature cycles on the system controller with a minimum storage capacity a four-dimensional frequency distribution should be used [7]. Figure 23 outlines the storage of $n_{Z}$ cycles with similar amplitudes $\Delta T_{J}$ and minimum temperatures $T_{J \text {,min }}$. To consider cycle specific heating times, each temperature class is linked with a histogram containing the number $n_{P}$ of cycles with equal time stamps. In Figure 23 the class width $T_{\mathrm{CW}}$ to group cycles with similar amplitudes is set to $T_{\mathrm{CW}}$ $=5^{\circ} \mathrm{C}$. To reach a compromise between the required storage capacity and the achievable accuracy in lifetime calculation, the resolutions of the temperature and the time axis were varied.

As an example of this variation Figure 24 diagrams the storage capacity $S$ and the lifetime calculation error $E$ in dependency of an increasing class width $T_{\mathrm{CW}}$ to group cycles with similar amplitudes $\Delta T_{J}$. It turns out that a class width of $T_{\mathrm{CW}}=5^{\circ} \mathrm{C}$ enables a reduction of the storage capacity to 
TABLE 1: Number of extreme values $N_{E}$ in the working storage during the analysis of four exemplary temperature profiles with an online Rainflow.

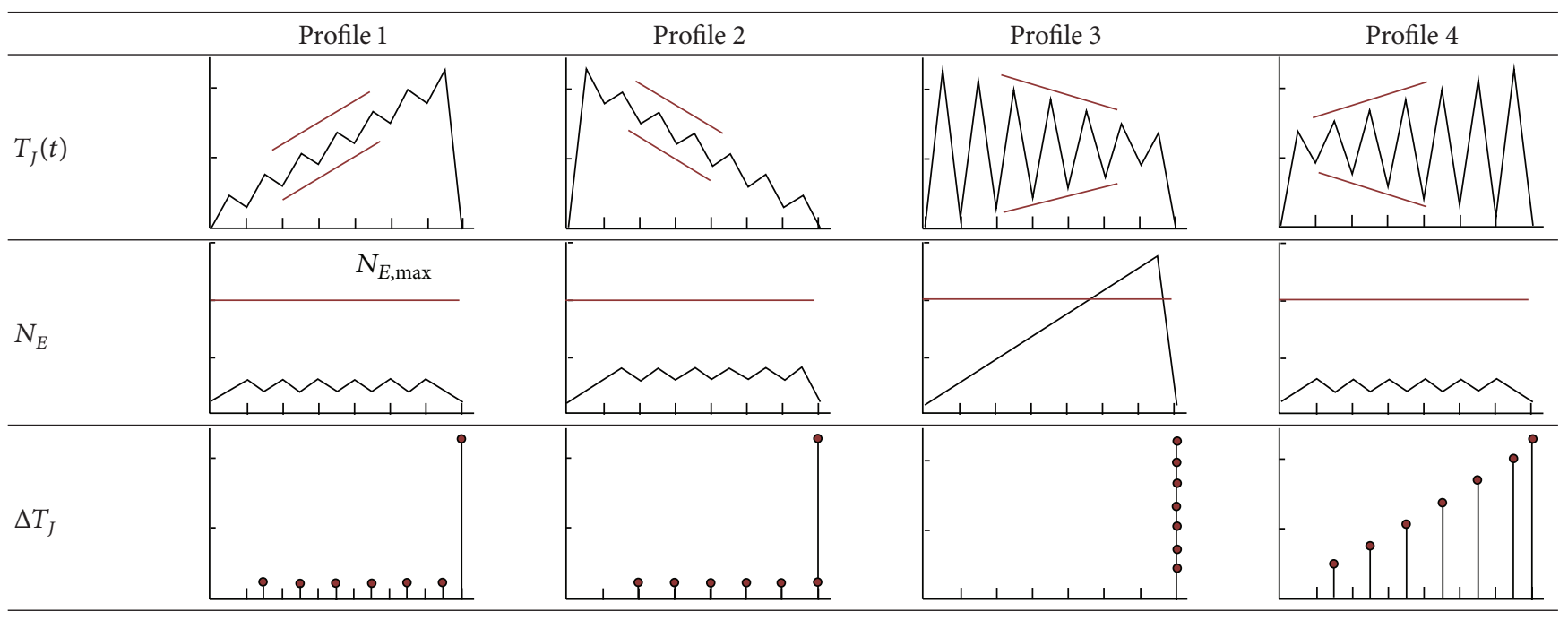

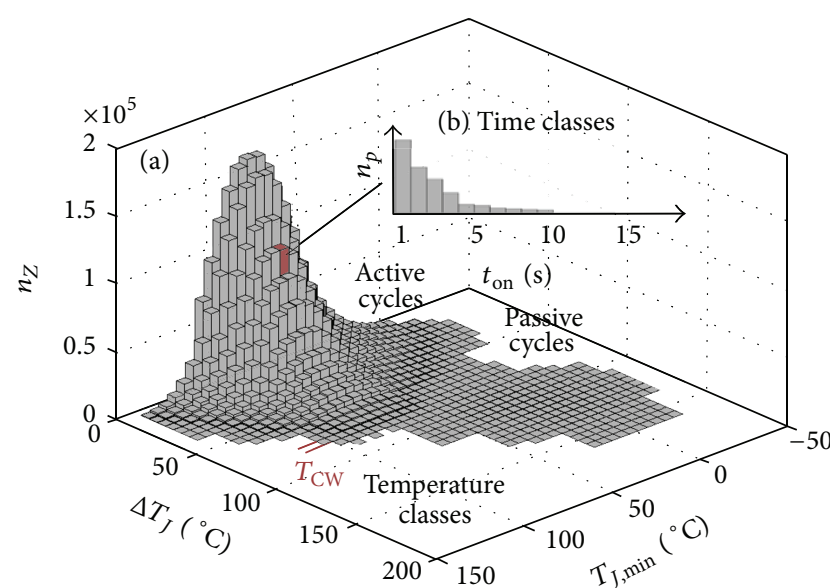

FIGURE 23: Storage of temperature cycles with similar (a) amplitudes $\Delta T_{J}$ and minimum cycle temperatures $T_{J \text {,min }}$ in a matrix. (b) Each matrix element contains a histogram that gives information about the cycle heating times $t_{\text {on }}$.

$S=51.6$ kilobytes. A further increase of the class width would lead to an increased error, which is caused by the temperature cycles that were rounded up into the next higher temperature class and their nonlinear overvaluing in the CIPS08 lifetime model. In the case of a class width of $T_{\mathrm{CW}}=5^{\circ} \mathrm{C}$ the calculated lifetime is affected by less than $E=-0.5$ percent, so that it is a suitable compromise between a minimum storage capacity and a good storage accuracy.

\subsubsection{Validation of the Online Cycle Recording Algorithm.} Finally the properties of the developted online cycle recording algorithm should be evaluated. The major advantage of a conventional algorithm, which was presented before, is that each temperature cycle becomes valued with its exact individual cycle parameters. For this reason a conventional algorithm enables the most accurate lifetime calculation, so

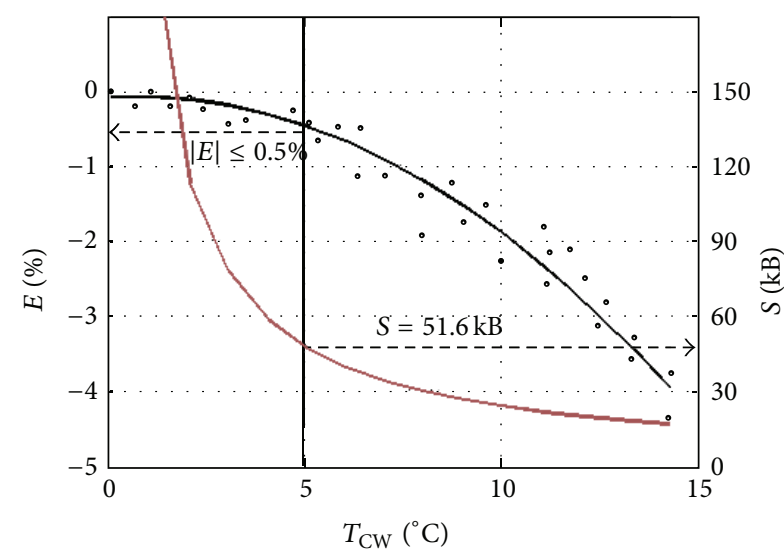

FIGURE 24: Required storage capacity $S$ and the error $E$ in the calculated module lifetime in dependency of an increasing class width $T_{\mathrm{CW}}$.

that it can be used as reference. In Figure 25 the required computing power to determine temperature cycles in the load-profile of a hybrid car and the required storage capacity to store them for lifetime calculation are diagrammed for the conventional algorithm and the developed online cycle recording algorithm.

It turns out that the required computing power to calculate the temperature cycles within the presented load-profile could be reduced by 85 percent due to an extreme value filter algorithm. Moreover the storage of the determined cycles in a four-dimensional frequency distribution and the variation of the class widths enable a reduction of the required storage capacity by 99 percent compared to minimum class widths of one degree and one second. Despite these major reductions the calculated lifetime on the basis of the temperature cycles determined by the online algorithm deviates less than 0.8 percent from the lifetime calculated with the conventional extracted cycles. Because of its properties the developed 


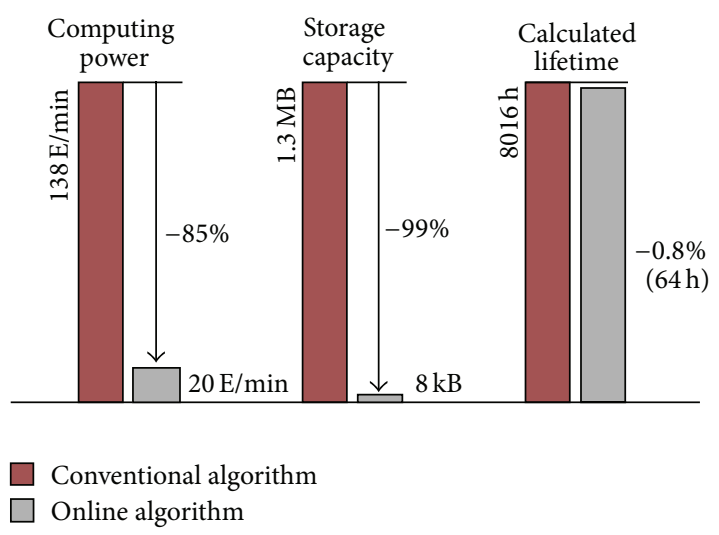

Figure 25: Comparison of the conventional algorithm used on desktop computers with the developed online cycle recording algorithm that should be implemented on the system controller of the voltage source inverter.

algorithm can be implemented on the system controller of the voltage source inverter to record the temperature cycles of the IGBT power module during its operation [24].

\section{Conclusions}

The analysis of the state of the art to design the lifetime of IGBT power modules used in hybrid cars showed that there is a need to verify the theoretical load-profiles with data from the field. For this purpose a temperature cycle recorder was presented that records the temperature cycles of an IGBT power module during its operation in a voltage source inverter. For the measuring of the junction temperature during inverter operation a modified IGBT gate driver was presented. The driver concept determines the temperature sensitive IGBT internal gate resistor by superimposing the negative gate voltage with a high-frequency identification signal. For this purpose the conventional IGBT driver was extended by an auxiliary MOSFET and a control unit that manages the feeding-in process during the regular switching operation, the transmission of the measured junction temperature, and the automatic calibration of the sensor system. Finally, it is shown that the driver enables realtime junction temperature measurement during the regular inverter operation.

In order to reduce the quantity of data that has to be stored on the system controller an online cycle recording algorithm was developed that processes the measured junction temperature into a frequency distribution of temperature cycles. A review of empirical lifetime models showed that each temperature cycle has to be parameterized with its amplitude, its minimum temperature, and its heating time. The algorithm consists of a filter that identifies all extreme values that are relevant for the lifetime calculation. An online rangecounting Rainflow algorithm processes them into closed temperature cycles and stores them in a four-dimensional frequency distribution. In relation to the cycle counting algorithm used on desktop computers the computing power could be decreased by 85 percent and the required storage capacity could be decreased to 8 kilobytes. Despite these reductions the compliance of the online algorithm and the conventional desktop computer algorithm amounts to $99 \%$.

Currently the IGBT driver with junction temperature measurement and the online temperature cycle recording algorithm are integrated in the voltage source inverter of first test vehicles. On the basis of the recorded load-histories it will be possible to verify and adjust the theoretical load-profiles used in today's lifetime calculation approach. For this reason the presented temperature cycle recorder makes an important contribution to improve the accuracy of the lifetime calculation of IGBT power modules and the reliability of future voltage source inverters.

\section{Conflict of Interests}

The authors declare that there is no conflict of interests regarding the publication of this paper.

\section{Acknowledgments}

This project was supported by the ZF Friedrichshafen AG. Special thanks go to the Department of Engineering Electric Mobility \& Mechatronics, Auerbach i. d. Opf.

\section{References}

[1] W. Sch, A. Hensler, J. Lutz et al., "Hybrid drive as a variation of a gear box," in Symposium Hybrid- und Elektrofahrzeuge, Braunschweig, Germany, February 2011.

[2] M. Ciappa, "Lifetime modeling and prediction of power devices," in Proceedings of the 5th International Conference on Integrated Power Systems (CIPS '08), pp. 27-36, 2008.

[3] M. Thoben, K. Mainka, R. Bayerer, I. Graf, and M. Münzer, "From vehicle drive cycle to reliability testing of power modules for hybrid vehicle inverters," in Proceedings of the International Exhibition and Conference for Power Electronics, Intelligent Motion and Power Quality (PCIM Europe '08), pp. 651-656, May 2008.

[4] J. Lutz, H. Schlangenotto, U. Scheuermann, and R. D. Doncker, Semiconductor Power Devices, Springer, New York, NY, USA, 2011.

[5] M. Denk and M.-M. Bakran, "An IGBT driver concept with integrated real-time junction temperature measurement," in Proceedings of the International Exhibition and Conference for Power Electronics, Intelligent Motion, Renewable Energy and Energy Management (PCIM '14), pp. 1-8, May 2014.

[6] M. Denk and M.-M. Bakran, "Comparison of counting algorithms and empiric lifetime models to analyze the load-profile of an IGBT power module in a hybrid car," in Proceedings of the 3rd International Electric Drives Production Conference (EDPC '13), October 2013.

[7] M. Denk and M.-M. Bakran, "Efficient online-algorithm for the temperature cycle recording of an IGBT power module in a hybrid car during inverter operation," in Proceedings of the 8th International Conference on Integrated Power Systems (CIPS '14), pp. 1-6, 2014.

[8] M. Ciappa, "Selected failure mechanisms of modern power modules," Microelectronics Reliability, vol. 42, no. 4-5, pp. 653667, 2002. 
[9] M. Held, P. Jacob, G. Nicoletti, P. Scacco, and M.-H. Poech, "Fast power cycling test for IGBT modules in traction application," in Proceedings of the 2nd International Conference on Power Electronics and Drive Systems (PEDS '97), pp. 425-430, May 1997.

[10] R. Bayerer, T. Herrmann, T. Licht, J. Lutz, and M. Feller, "Model for power cycling lifetime of IGBT modules-various factors influencing lifetime," in Proceedings of the 5th International Conference on Integrated Power Systems (CIPS '08), pp. 37-42, Nuremberg, Germany, March 2008.

[11] Y. Wang, S. Jones, D. Chamund, and G. Liu, Lifetime Modelling of IGBT Modules Subjected to Power Cycling Tests, PCIM, 2013.

[12] J. Lutz, "IGBT-modules: design for reliability," in Proceedings of the 13th European Conference on Power Electronics and Applications (EPE '09), pp. 1-3, Chemnitz University of Technology, 2009.

[13] K. Mainka, M. Thoben, and O. Schilling, "Lifetime calculation for power modules, application and theory of models and counting methods," in Proceedings of the 14th European Conference on Power Electronics and Applications (EPE '11), pp. 1-8, IEEE, Birmingham, UK, September 2011.

[14] M. Ciappa, F. Carbognani, and W. Fichtner, "Lifetime prediction and design of reliability tests for high-power devices in automotive applications," IEEE Transactions on Device and Materials Reliability, vol. 3, no. 4, pp. 191-196, 2003.

[15] F. Ellyin, Fatigue Damage, Crack Growth and Life Prediction, Chapman \& Hall, New York, NY, USA, 1996.

[16] ASTM E-1049, "Standard Practices for Cycle Counting in Fatigue Analysis," Annual Book of ASTM Standards, 1999.

[17] M. Miner, "Cumulative damage in fatigue," Jornal of Applied Mechanics, vol. 12, pp. 159-164, 1945.

[18] E. R. Motto and J. F. Donlon, "IGBT module with user accessible on-chip current and temperature sensors," in Proceedings of the 27th Annual IEEE Applied Power Electronics Conference and Exposition (APEC '12), pp. 176-181, Orlando, Fla, USA, February 2012.

[19] D. Wagenitz, A. Hambrecht, and S. Dieckerhoff, "Lifetime evaluation of IGBT power modules applying a nonlinear saturation voltage observer," in Proceedings of the 7th International Conference on Integrated Power Electronics Systems (CIPS '12), pp. 256-260, March 2012.

[20] B. Ji, V. Pickert, and B. Zahawi, "In-situ bond wire and solder layer health monitoring circuit for IGBT power modules," in Proceedings of the 7th International Conference on Integrated Power Electronics Systems (CIPS '12), March 2012.

[21] F. Richardeau, M. Morvan, and F. Mosser, On-Line TJ Monitoring Sensor Embedded on VSI Driver Board Application on a $5 \mathrm{~kW}$ High Speed PMSM for Aeronatic Blowing-Fan, PCIM, 2014.

[22] I. Bahun, V. Sunde, and Z. Jakopovic, "Estimation of insulatedgate bipolar transistor operating temperature: simulation and experiment," Journal of Power Electronics, vol. 13, no. 4, pp. 729736, 2013.

[23] V.-K. Sundaramoorty, E. Bianda, R. Bloch, and F. Zurfluh, "Simultaneous online estimation of junction temperature and current of IGBTs using emitter-auxiliary emitter parasitic inductance," in Proceedings of International Exhibition and Conference for Power Electronics, Intelligent Motion, Renewable Energy and Energy Management (PCIM '14), pp. 1-8, Nuremberg, Germany, 2014.

[24] H. Kuhn and A. Mertens, "On-line junction temperature measurement of IGBTs based on temperature sensitive electrical parameters," in Proceedings of the 13th European Conference on Power Electronics and Applications (EPE '09), September 2009.

[25] D. Barlini, M. Ciappa, A. Castellazzi, M. Mermet-Guyennet, and W. Fichtner, "New technique for the measurement of the static and of the transient junction temperature in IGBT devices under operating conditions," Microelectronics Reliability, vol. 46, no. 9-11, pp. 1772-1777, 2006.

[26] V. Sundaramoorthy, E. Bianda, R. Bloch, I. Nistor, G. Knapp, and A. Heinemann, "Online estimation of IGBT junction temperature (Tj) using gate-emitter voltage (Vge) at turn-off," in Proceedings of the 15th European Conference on Power Electronics and Applications (EPE '13), September 2013.

[27] W. Brekel, T. Dutemeyer, G. Puk, and O. Schilling, Time Resolved In Situ $T_{v j}$ Measurements of $6.5 \mathrm{kV}$ IGBTs during Inverter Operation, PCIM, 2009.

[28] Infineon Technologies, "Infineon technical information," Tech. Rep. FF225R12ME4, Infineon Technologies, 2013.

[29] Infineon Technologies, "Industrial IGBT modules, explanation of technical information," Application Note AN 2011-05, 2013, http://www.infineon.com/.

[30] E. Hoene and T. Baumann, "Device for measuring a temperature of a power semiconductor," WO2013/000971, 2013.

[31] R. Schmidt and U. Scheuermann, "Using the chip as a temperature sensor-the influence of steep lateral temperature gradients on the Vce(T)-measurement," in Proceedings of the 13th European Conference on Power Electronics and Applications (EPE '09), September 2009.

[32] L. Gopireddy, L. M. Tolbert, and B. Ozpineci, "Lifetime prediction of IGBT in a STATCOM using modified-graphical rainflow counting algorithm," in Proceedings of the 38th Annual Conference on IEEE Industrial Electronics Society (IECON '12), pp. 3425-3430, October 2012.

[33] G. Glinka and J. C. P. Kam, "Rainflow counting algorithm for very long stress histories," International Journal of Fatigue, vol. 9, no. 4, pp. 223-228, 1987.

[34] S. D. Downing and D. F. Socie, "Simple rainflow counting algorithms," International Journal of Fatigue, vol. 4, no. 1, pp. 3140, 1982.

[35] R. Krümmer, R. Teimann, G. Berger, J. Petzoldt, and L. Lorenz, "On-line calculation of the chip temperature of power modules in voltage source converters using the microcontroller," in Proceedings of the 8th European Conference on Power Electronics and Applications (EPE '99), Lausanne, Switzerland, 1999. 

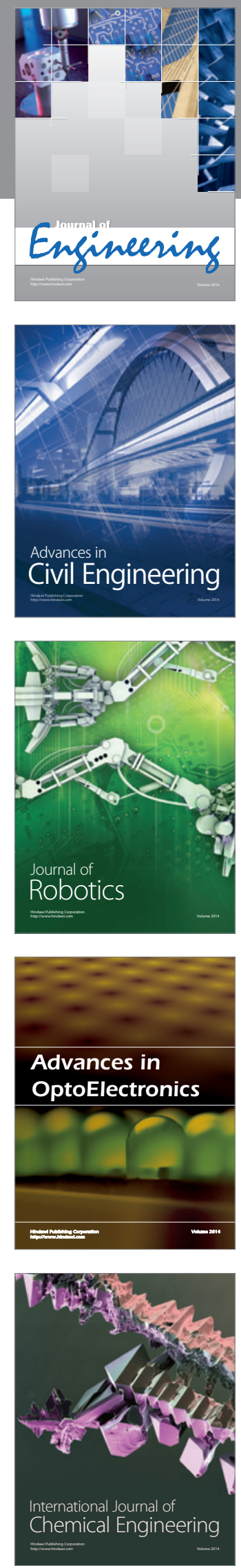

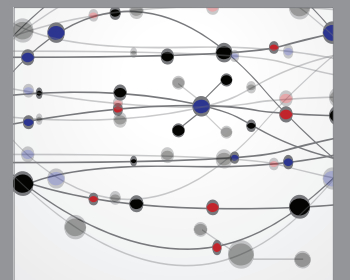

The Scientific World Journal
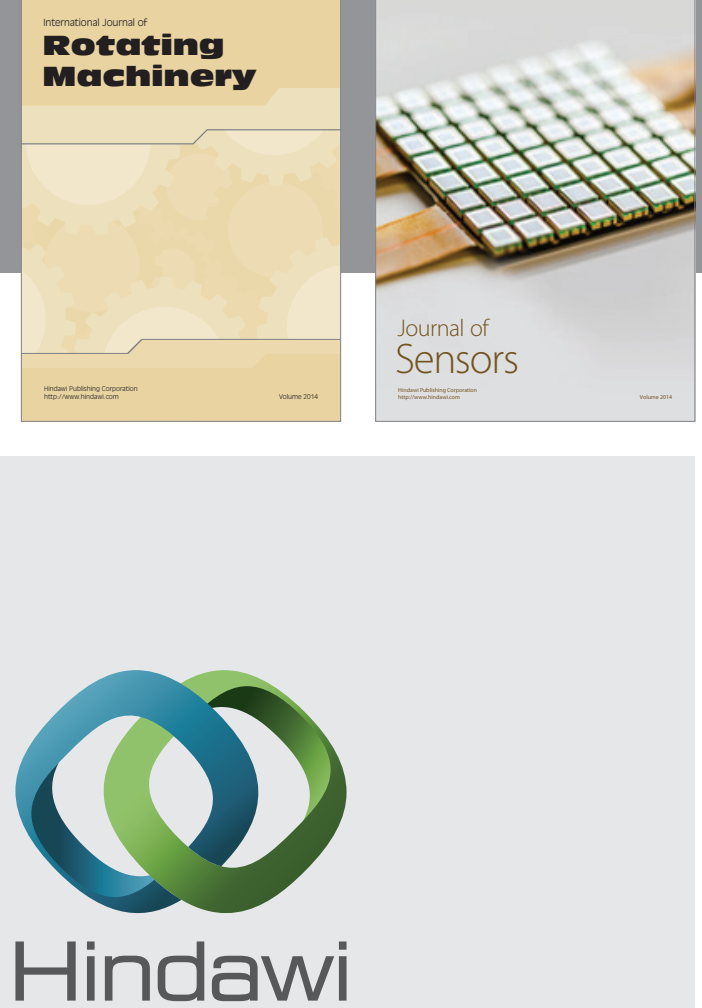

Submit your manuscripts at http://www.hindawi.com
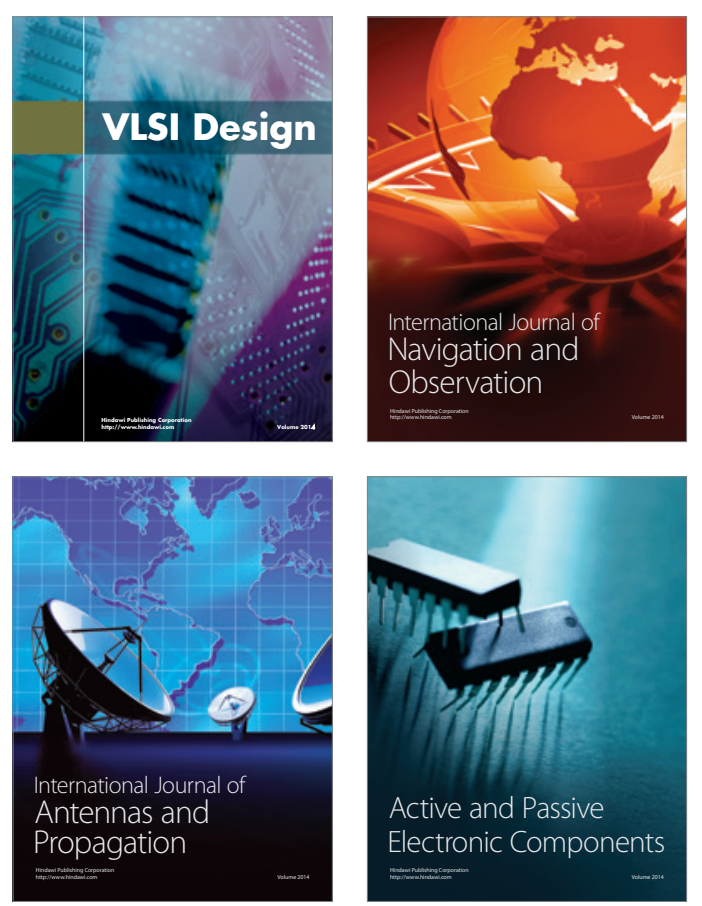
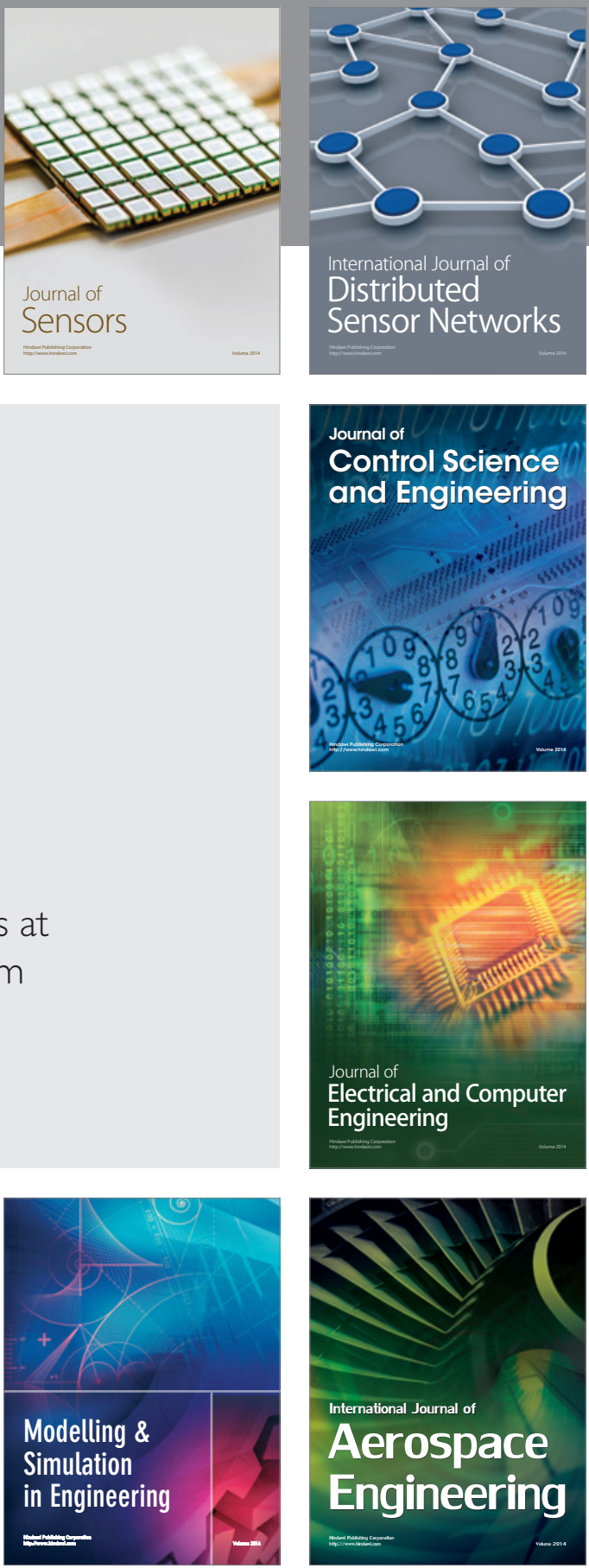

Journal of

Control Science

and Engineering
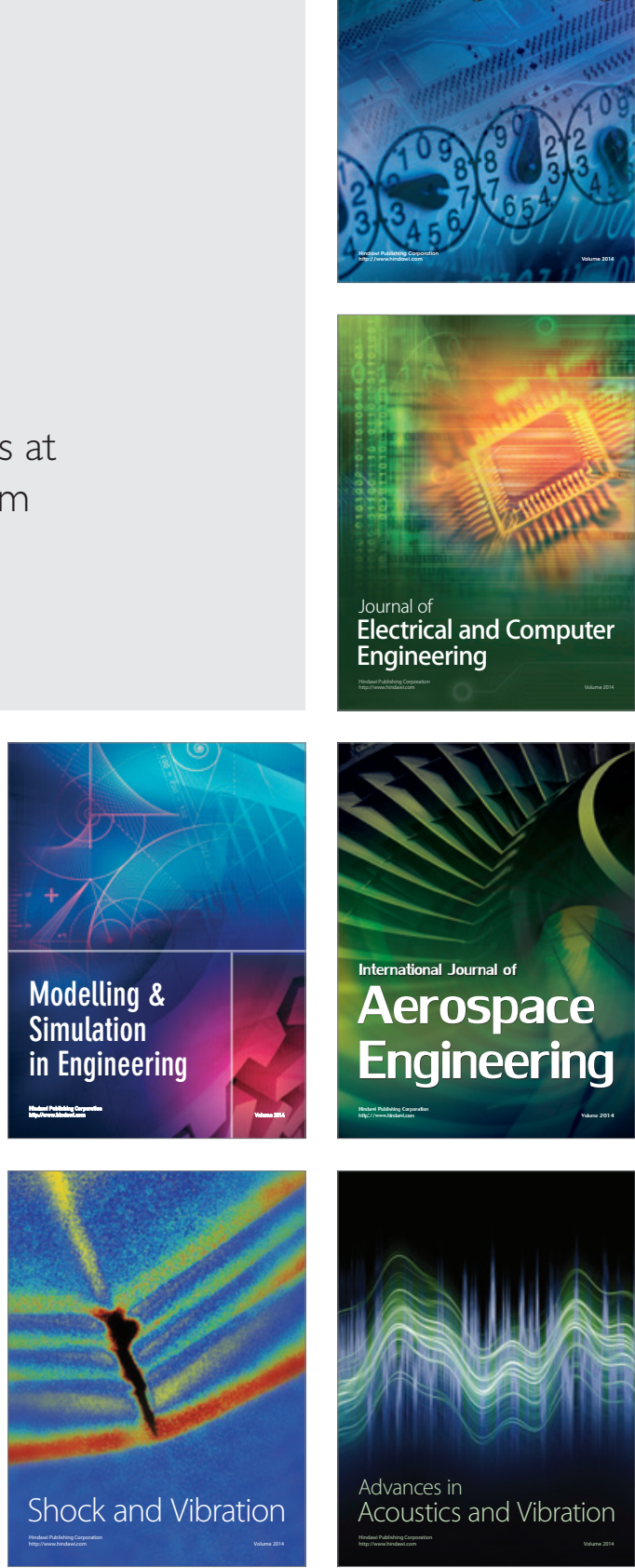